\title{
Correction to: Gender and Binegativity: Men's and Women's Attitudes Toward Male and Female Bisexuals
}

\author{
Megan R. Yost ${ }^{1,3} \cdot$ Genéa D. Thomas $^{2}$
}

Published online: 2 February 2022

(c) Springer Science+Business Media, LLC, part of Springer Nature 2022

\section{Correction to: Arch Sex Behav (2012) 41:691-702 https://doi.org/10.1007/s10508-011-9767-8}

In Table 3 (p. 698), the row referencing "Wanting attention" incorrectly reported values for "female bisexual targets" in the columns for "male bisexual targets," and vice versa. The authors would like to thank Dr. Alan Smerbeck at the Rochester Institute of Technology for alerting us to this error.

Two rounding errors in percentages were also identified and have been corrected: male bisexual target, "gender nonconforming," total (all raters) and female bisexual target, "really gay," male rater.

Table 3 needs to be corrected to:

The original article can be found online at https://doi.org/10.1007/ s10508-011-9767-8.

Megan R. Yost

yostm@dickinson.edu

1 Departments of Psychology and Women's, Gender and Sexuality Studies, Dickinson College, Carlisle, PA, USA

2 Department of Psychology, Dickinson College, Carlisle, PA, USA

3 Department of Psychology, Dickinson College, P.O. Box 1773, Carlisle, PA 17013, USA 
Table 3 Thematic analysis of open-ended items, separated by target's and rater's sex

\begin{tabular}{|c|c|c|c|c|c|c|}
\hline \multirow[t]{2}{*}{ Theme } & \multicolumn{3}{|c|}{ Male bisexual target } & \multicolumn{3}{|c|}{ Female bisexual target } \\
\hline & Male rater & Female rater & Total (all raters) & Male rater & Female rater & Total (all raters) \\
\hline Definition & $35(41 \%)$ & $101(62 \%)$ & $136(55 \%)$ & $36(41 \%)$ & $108(66 \%)$ & $144(57 \%)$ \\
\hline Really heterosexual & $1(1 \%)$ & $1(1 \%)$ & $2(1 \%)$ & $5(6 \%)$ & $9(6 \%)$ & $14(6 \%)$ \\
\hline Really gay & $18(21 \%)$ & $25(15 \%)$ & $43(17 \%)$ & $3(3 \%)$ & $2(1 \%)$ & $5(2 \%)$ \\
\hline Wanting attention & 0 & 0 & 0 & $3(3 \%)$ & $9(6 \%)$ & $12(5 \%)$ \\
\hline Gender non-conforming & $13(15 \%)$ & $29(18 \%)$ & $42(17 \%)$ & $5(6 \%)$ & $3(2 \%)$ & $8(3 \%)$ \\
\hline Liberal and open & $8(9 \%)$ & $13(8 \%)$ & $21(8 \%)$ & $16(18 \%)$ & $25(15 \%)$ & $41(16 \%)$ \\
\hline Sexy & 0 & 0 & 0 & $10(11 \%)$ & $6(4 \%)$ & $16(6 \%)$ \\
\hline Negative & $10(12 \%)$ & $5(3 \%)$ & $15(6 \%)$ & $5(6 \%)$ & $2(1 \%)$ & $7(3 \%)$ \\
\hline
\end{tabular}

Number of participants is provided first, followed by percentage in parentheses

Publisher's Note Springer Nature remains neutral with regard to jurisdictional claims in published maps and institutional affiliations. 